\title{
Experimental set-up MARUSYA-FLINT for the study of correlations in cumulative process at Nuclotron- M beams. Current status.
}

Baldin A.A. a, Chernyshov O.A. ${ }^{b}$, Goryachev V.S. ${ }^{b}$, Zhigareva N.M. ${ }^{a, b}$, Kirin D.Yu. ${ }^{b}$, Konstantinov A.V. ${ }^{a}$, Kudashkin I.V. ${ }^{a}$, Malahov A.I. ${ }^{a}$, Mikhailov K.R. ${ }^{a, b}$, Polozov P.A. ${ }^{\text {b }}$, Prokudin M.S. ${ }^{b}$, Romanov D.V. ${ }^{\text {}}{ }^{1}$, Stavinskiy A.V. ${ }^{b}$, Stolin V.L. ${ }^{\text {b }}$, Sharkov G.B. ${ }^{b}$, Shimansky S.S. ${ }^{a}$

a Joint Institute for Nuclear Research

141980, Joliot-Curie 6, Dubna, Moscow region, Russia

${ }^{b}$ Institute for Theoretical and Experimental Physics

117218, B.Cheremushkinskaya 25, Moscow, Russia

E-mail: romanoveitep.ru

Abstract

The experimental program for the study of properties of cold superdense matter is proposed for NUCLOTRON accelerator at the Joint Institute for Nuclear Research in Dubna. The region of phase diagram of nuclear matter with temperature and baryon density typical for neutron stars will be studied at the laboratory. Experimental setup for this program is created on the basis of synthesis experiments MARUSYA (JINR) and Flint (ITEP).

XXII International Baldin Seminar on High Energy Physics Problems

15-20 September 2014

JINR, Dubna, Russia

${ }^{1}$ Speaker 


\section{Introduction}

The aim of the experiment is the study of properties of cold superdense matter [1]. In this experiment we propose to investigate the region of the phase diagram of nuclear matter, corresponding to low temperatures and high baryon density (Figure 1)

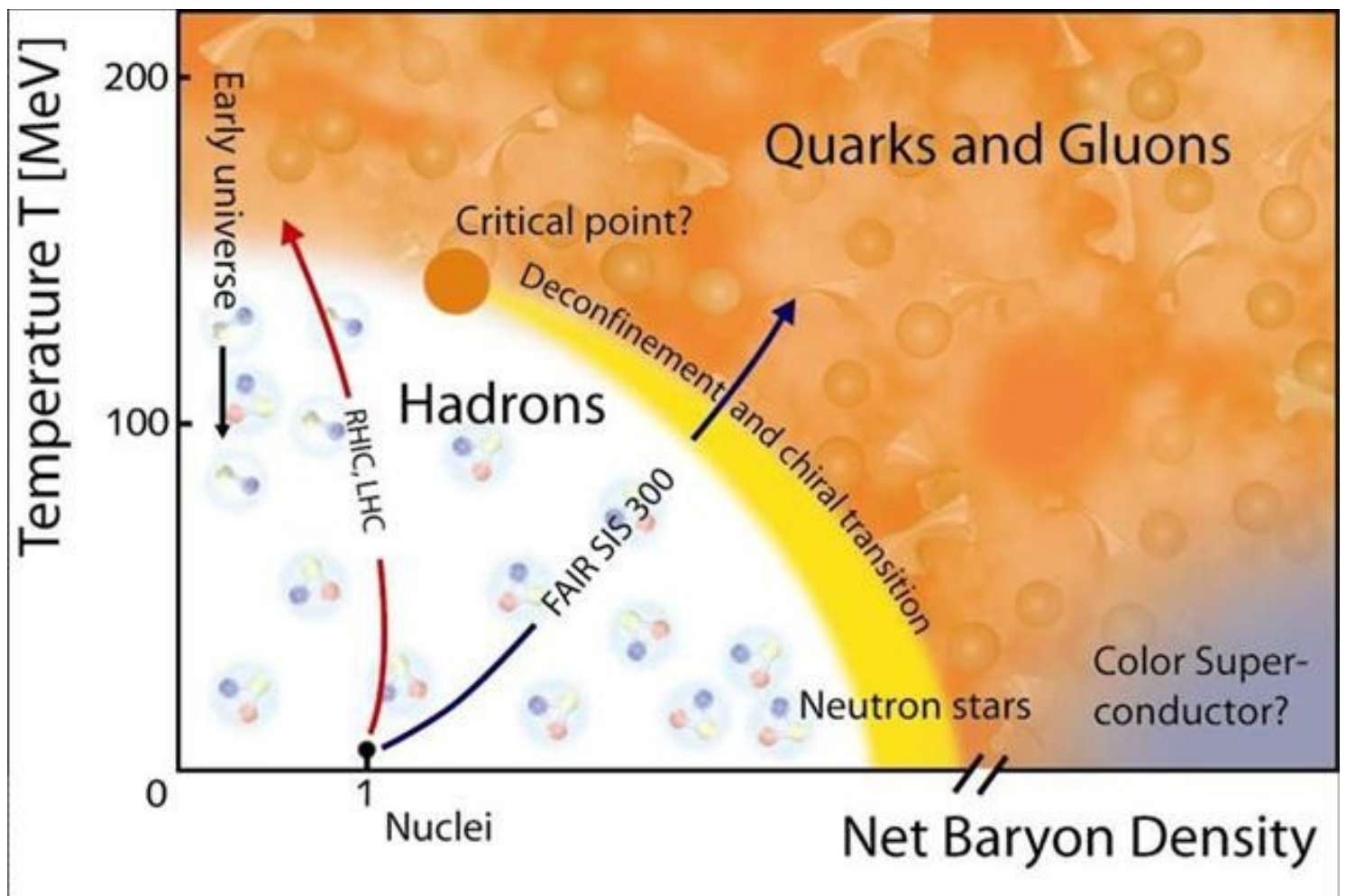

Figure 1. Phase diagram of nuclear matter

Currently, there is great uncertainty in the theoretical predictions of the equation of state of nuclear matter at low temperatures and high densities. There are many theoretical models that try to describe the properties of this state of nuclear matter. The properties of cold superdense state of nuclear matter are of particular interest, because the similar conditions are expected for the matter at the center of massive stars and control their evolution. Up to now there are no experimental data on the properties of this state, obtained in laboratory experiments.

\subsection{Experimental program}

The main objectives of the experimental program are as follows:

1. To find optimal kinematical range for the study of cold superdense nuclear matter and to obtain information on the nature of cold and dense baryonic component in various nuclei. Determination of cold and dense baryonic fraction in ordinary nuclear matter.

2. To study the properties of superdense cold baryonic component using both the fragmentation and hard scattering mechanism of the cumulative particle production. 3 . The study of new states of matter in the density and temperature ranges, typical for neutron stars. 
Two-arms detector MARUSYA - FLINT is created on the base of two single-arm detectors FLINT (ITEP) and MARUSYA (JINR), which are briefly described in the following two paragraphs.

\subsection{FLINT}

The experimental apparatus FLINT for the study of cold dense matter were placed at the magnetic hall of ITEP accelerator complex TVN (U-10) [2].

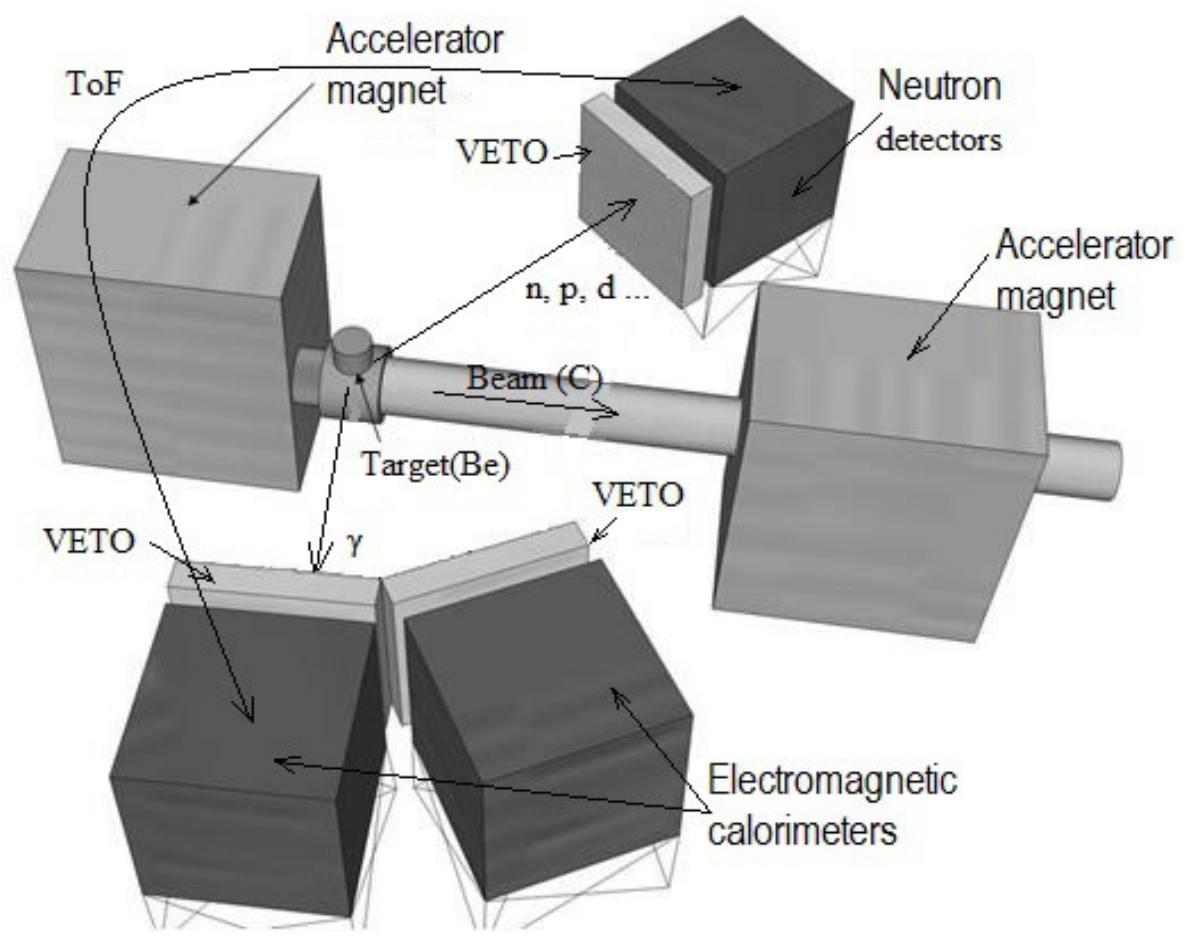

Figure 2. FLINT set-up (ITEP)

FLINT set-up includes (figure 2):

- two 64-channel electromagnetic calorimeter with threshold veto system [3]

- power supply

-data acquisition system

-control and monitoring system.

Selection of events, relevant interaction between two multinucleon fluctuations (fluctons) in the experiment FLINT realized by means of photon trigger with high transverse momentum in the central rapidity region.

Photons have been detected by two 64-channel lead glass calorimeters; the size of each calorimeter is $0.8 \times 0.8 \mathrm{~m}^{2}$. Each calorimeter channel was equipped with LED calibration system and threshold veto detector. VETO system consists of 64 independent scintillation detectors (thickness $5 \mathrm{~mm}$ ) with SPM, located at the centers of the respective blocks of lead glass. The 
size of each VETO counters and each calorimetric module is the same $\left(100 \times 100 \mathrm{~mm}^{2}\right)$. VETO system is used for identifying (if necessary - for rejection) of charged particles.

Further development of the experiment assumed the creation of the second arm with multichannel detector of nuclear fragments, including neutrons and protons [4]. At the time of the fire accident at the U-10 prototypes of the neutron detector modules were made and tested.

\subsection{MARUSYA-FLINT}

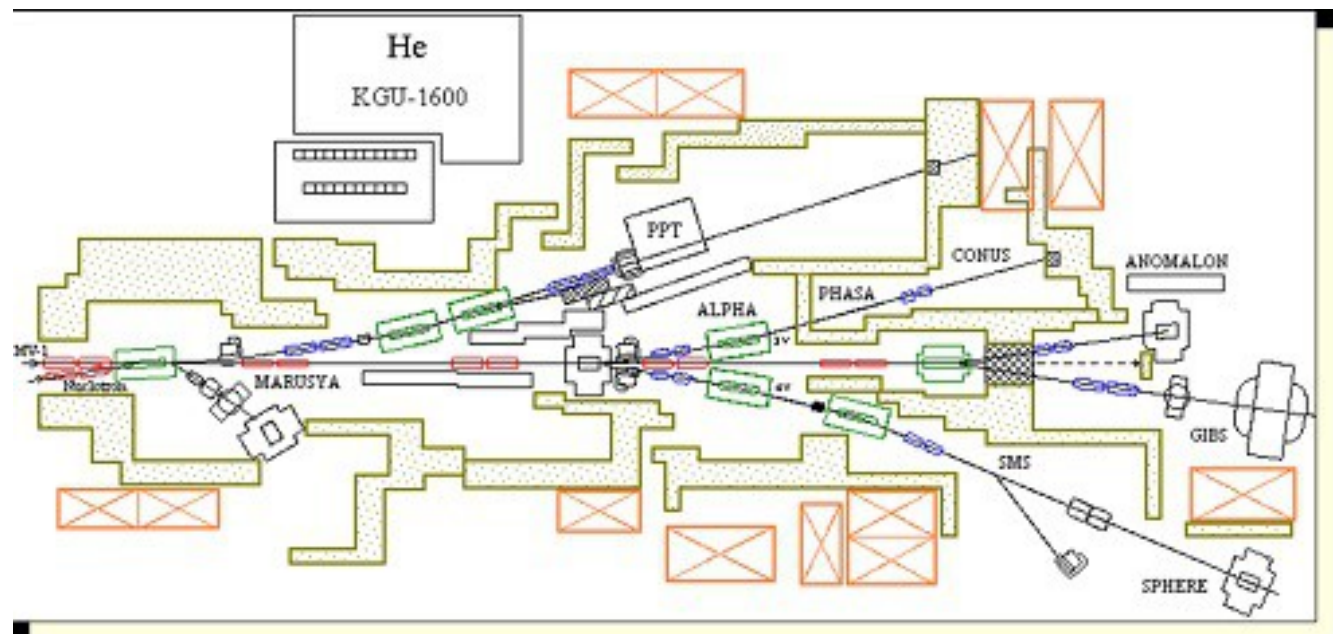

Figure 3. Scheme of the experimental hall

Location MARUSYA setup in the NUCLOTRON experimental hall is shown in Figure 3. MARUSYA with added FLINT detectors is shown in Figure 4.

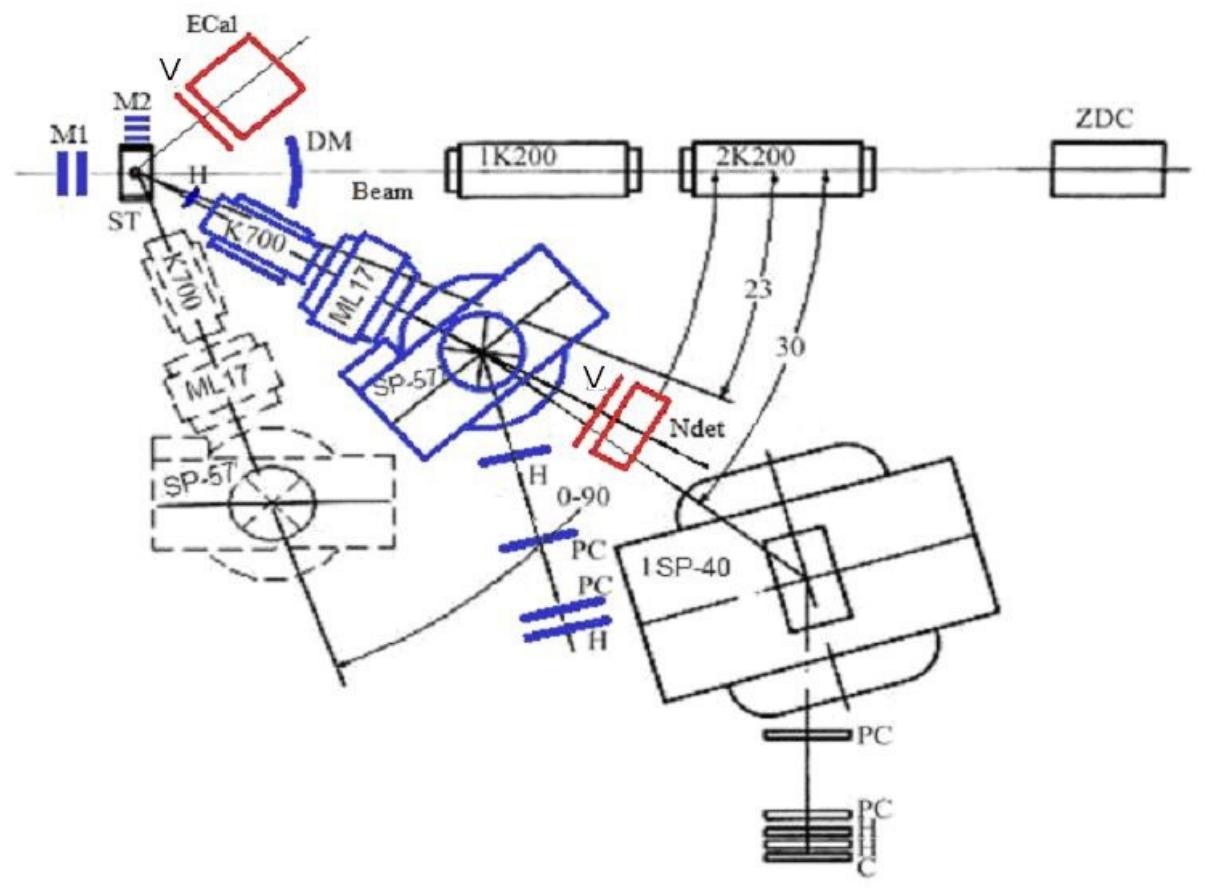

Figure 4. The experimental setup MARUSYA-FLINT. ST - target station, $\mathrm{M}_{1}, \mathrm{M}_{2}$ - monitor systems, DM- multiplicity detectors, H - scintillation hodoscopes, V - VETO systems for 
calorimeters and neutron detectors, ZDC - hadron calorimeter, PC - proportional chambers, C cherenkov counter, ML17, K100 - quadrupole lens, SP-57, SP-40 - dipole magnets, ECal electromagnetic calorimeter, Ndet- neutron detector.

MARUSYA setup [5] whose elements in Figure 4 are indicated in blue, includes: a target station, monitoring systems, multiplicity detector, scintillation hodoscopes, proportional chambers, Cherenkov counter, magnetic lenses and bending magnets. Polar angle of magnetic spectrometer can be changed from 23 to 90 degrees.

The spectrometer can be operated in two modes:

For particles identification within momentum range $0.3-0.8 \mathrm{GeV} / \mathrm{c}$ magnet SP-40 is not used. This dipole magnet is used for additional magnetic analyses within momentum range $0.8-2 \mathrm{GeV}$ /c. Coordinate information from scintillation hodoscopes is used for momentum calculation. Hodoscopes are placed before analyzing magnet and in its focal plane.

The system of coordinate detectors includes scintillation strips $1 \mathrm{~cm}$ wide and provides momentum resolution of $2-5 \%$ in the momentum range $0.6-2 \mathrm{GeV} / \mathrm{c}$.

Additional FLINT detectors (electromagnetic calorimeter, neutron detector, and VETO systems) is shown in Figure 4 in red.

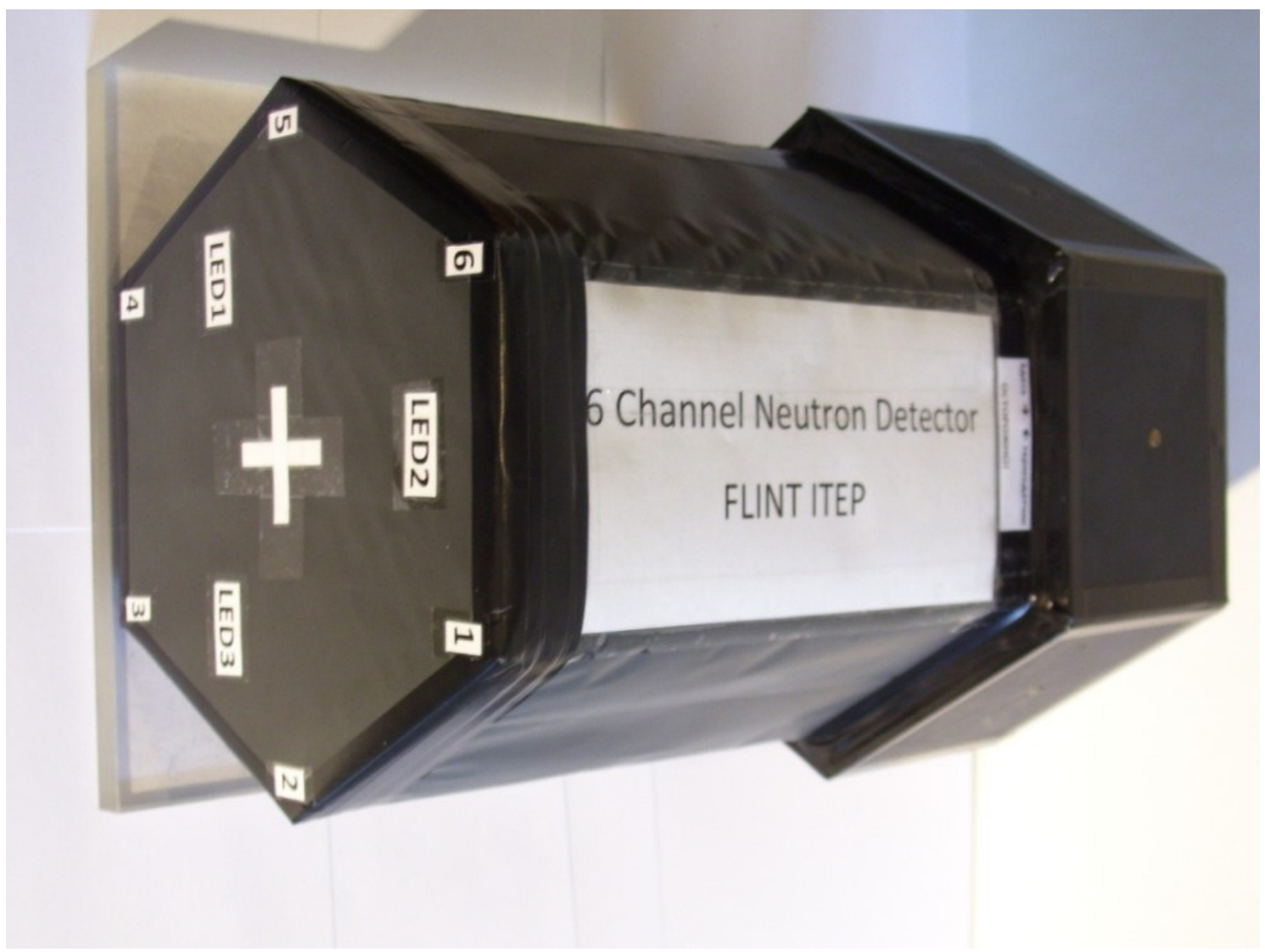

Figure 5. View of the neutron detector.

Nine-channels electromagnetic calorimeter was used in test experiments at the Nuclotron in late 2013 and early 2014. 9 channels provides the minimum configuration to restore the energy of one photon. 
Measurements were made in this configuration at different targets and nuclei. Data analysis is in progress.

\subsection{Conclusions}

Experimental setup MARUSYA-FLINT, which includes all necessary elements is created. Its commissioning is in progress.

The main present day objective is to combine MARUSYA and FLINT infrastructures, data acquisition systems (ADC VME chassis and server), and a power control systems. The next steps will be to test and hold calibrate Electromagnetic calorimeter with Nuclotron beams, to activate head portion of the rotating magneto-optical spectrometer MARUSYA including a set of time-of-flight scintillation detectors (right arm), to incorporate into the setup systems of FLINT VETO counters and FLINT neutron detector. Prototype of FLINT neutron detector is shown at Figure 5.

This work was supported by grants 14-02-00896_a, 14-02-93108-NCNIL_a

\section{References}

[1] S.S. Shimansky, in Proc. of the VIII Intern. Workshop on Relativistic Nuclear Physics:from Hundreds of MeV to TeV, May 23-28, 2005 , 297 (Dubna, 2006); nucl-ex/0604014; K.R. Mikhailov, A.V. Stavinskiy, V.L. Stolin, G.B. Sharkov «Exotica in dense and cold nuclear matter». Phys.Atom.Nucl. 77 (2014) 576-580 ; A. Stavinskiy «Dence Cold Matter» EPJ Web Conf. 71 (2014) 00125

[2] K. Mikhailov et al. «Double cumulative photon spectra at mid rapidity and high $p T$ in $C+B e$ collisions at 2.0 and 3.2 AGeV» PoS Baldin-ISHEPP-XXI (2012) 104; I.G. Alekseev et al., "FLINT setup for cumulative process study with photon production" PTE, 2008, No4, p.5-12

[3] I. Alekseev et al. «Lead glass calorimeter calibration (in Russian)», 2006, Preprint ITEP 20-06

[4] A.Stavinsky, K.Mikhailov "Position sensitive neutron detector." IV Cherenkov Readings. Moscow, Lebedev Physical Institute. April 12, 2011

[5] A. A. Baldin et al. «Measurement of Magnetic Field Map for Magnetooptical Spectrometer MARUSYA », JINR-communication, P13-2006-67. 